\title{
Pur-alpha participates in the progression of Alzheimer's disease through direct and indirect ways
}

\section{Xiaoguang Shi}

Ningxia Medical University

\section{Shuanglai Ren}

Ningxia Medical University

\section{Bingying Zhang}

Ningxia Medical University

\section{Shanshan Guo}

Ningxia Medical University

Wenxin He

Ningxia Medical University

Chengmin Yuan

Ningxia Medical University

\section{Xiaofan Yang}

Hongqi Hospital Affiliated to Mudanjiang Medical University

Kevin Ig-Izevbekhai

University of Pennsylvania Perelman School of Medicine

Tao Sun

Ningxia Medical University

\section{Qinwen Wang}

Ningbo University

Jianqi Cui ( $\nabla$ jianqi@gmail.com )

https://orcid.org/0000-0003-2838-7473

\section{Research article}

Keywords: Pur-alpha, Alzheimer's disease, neurodevelopment, Ab clearance, RNA-seq, ChIP-seq

Posted Date: March 31st, 2020

DOl: https://doi.org/10.21203/rs.3.rs-19973/v1

License: () (i) This work is licensed under a Creative Commons Attribution 4.0 International License. Read Full License 


\section{Abstract}

Background: Purine rich element binding protein A (Pur-alpha)『encoded by the PURA gene区 is an important transcriptional regulator that binds to DNA and RNA and is involved in processes such as DNA replication and RNA translation. Pur-alpha plays an important role in the nervous system. Our previous research found that the regulatory effect of Pur-alpha on APP suggests that it may be involved in the production of betaamyloids. Suggestting that Pur-alpha may plays a role in Alzheimer's disease (AD), but the relevant evidence is insufficient.

Methods: We performed RNA-sequencing (RNA-seq) analysis of Pura-KO mouse hippocampal neuronal cell line (HT22) to analyze the effect of puralpha deletion on neuron expression profile. And then combined with ChIP-seq analysis to explore the mechanism of Pura on gene regulation.

Results: we found 656 differentially expressed genes between HT22 and Pura-KO HT22. A total of 62 overlapping genes were found by comparison with early brain protein expression profiles in mice, which may play important role in early neuronal development. We found 7 Alzheimer's disease (AD)-related genes (Lpl, Mapt, Mme, Ndufa3, Lrp1, Gapdh, mt-Co3) and 5 Ab clearance related genes (Mme, C3, Lrp1, Insr, Trem2) were regulated by Pur-alpha, suggesting that Pur-alpha plays an important role in AD. Through ChIP-seq analysis, we found that Pur-alpha binds directly to 47 genes and regulates their transcription, including Insr, Mapt, VIdlr, Jag1, etc. The direct regulation of Vldlr囚the Reelin ligand囚suggests that Pur-alpha may be involved in the process of synaptic plasticity. The direct regulation of Jag1 suggests that Pur-alpha may be involved in the Notch pathway.

Conclusions: Our study re-confirms the important role of Pur-alpha in neurodevelopment. The regulation of Pur-alpha on AD-related genes means that Pur-alpha plays an important role in the pathogenesis of AD.

\section{Background}

Pura is a 322-amino acid protein encoded by PURA, presenting mainly in the nucleus and minor in cytoplasm and mitochondria [1]. It can bind to purine rich DNA or RNA, and promoter regions of some genes in order to form multimeric complexes, and can also interact with other transcription factors [2]. Pur-alpha's function is complex-it can promote transcription of some genes, such as TNF-a [3], myelin basic protein [4], placental lactogen [5], and PDGF- Protein A [6], etc. At the same time, Pur-alpha also inhibits the expression of some genes, such as fas [7], $\alpha$-actin [8], amyloid- $\beta$ protein precursor[9] and CD43 [10].

Pur-alpha is widely expressed in the cerebellum, adrenal glands, and other tissues [11],suggesting that it has a wide range of effects throughout the human body. Pur-alpha plays a major role in the development and maintenance of the nervous system [12]. Mice lacking the Pura gene developed severe tremors and spontaneous seizures 2 weeks after birth and died at about 4 weeks of age [13].

In the past few years, we have focused on the role of Pur-alpha in the nervous system, including repairing DNA damage in neurons[14] and influencing Alzheimer's disease pathogenesis[15]. Recent technological developments in the life sciences research have allowed new insights into the functions of Pur-alpha. In our 
study, we performed RNA-seq and ChIP-seq analysis on Pura knockout cell lines constructed based on CRISPR/Cas 9 gene editing technology. We hope to improve the understanding of Pur-alpha through these investigations.

\section{Methods}

\section{Cell culture}

The HT22 cell line was maintained in the laboratory. The Pura-KO cell line was independently constructed and validated by the laboratory, based on CRISPR/Cas9, and screened for single cell cultured monoclonal cell lines. The cells were cultured in an incubator at $37^{\circ} \mathrm{C}$. Nutrient composition: 89\% DMEM (Bioind, USA), 10\% FBS (Bioind, USA) and 1\% penicillin-streptomycin (Solarbio, China).

\section{RNA-seq}

Whole genome RNA was extracted using the TRIZOL method, RNA concentration was determined using NanoDrop 2000 (Thermo), and RNA integrity was detected using an Agilent Bioanalyzer 2100 system (Agilent Technologies, CA, USA). The sequencing library was built using the NEBNext UltraTM RNA Library Preparation Kit for Illumina (NEB, USA) and library quality was assessed on an Agilent Bioanalyzer 2100 system. Clustering was performed using the TruSeq PE Cluster Kit v4-cBot-HS (Illumia), after which the library preparations were sequenced on an Illumina Hiseq Xten platform and the readings at the paired ends were generated.

ChIP-seq

ChIP immunoprecipitation was performed using a PierceTM Agarose ChIP Kit (Catalog number: 26156, Thermo USA). The antibody used to capture Pur-alpha was purchased from SANTA CRUZ (sc-130397). HT22 cells were seeded in a $10 \mathrm{~cm}$ diameter petri dish and subjected to ChIP immunoprecipitation with cell growth to $95 \%$ confluence. The ChIP immunoprecipitation operation was carried out according to the instructions. The main processes included formaldehyde cross-linking, sonication, enrichment of the target protein with the magnetic beads coated with the antibody, de-crosslinking, and recovery of the DNA fragment. The library was constructed using the ChIP-seq Library Prep Master Mix Set for Illumina (NEB, USA), the main processes including end repair, add in $A$ to the 3 'end, ligate adaptation, gel purification and size selection, PCR amplification. Sequencing was performed using the Illumina HiSeq 2500 sequencing platform.

Sequencing data quality control

Raw data in the fastq format (original reads) removed the purchase of ploy- $\mathrm{N}$ and low-quality reads to get clean data (clean reads). At the same time, Q20, Q30, GC content and sequence repeat levels of clean data were calculated (S5). All downstream analyses are based on high quality, clean data.

Differential gene acquisition 
The sequencing fastq data were compared to the mouse reference gene (GRCm38/mm10) using hisat2. The samtools tool was used to convert the obtained sam file to a bam file. The readings were counted using the htseq-count tool. The read matrices were analyzed using the edgeR tool to obtain differential genes with a fold multiple of $\geq 2$ and FDR $<0.05$ as a screening criterion. Since no biological duplication was set in this study, CORNAS (overlying RNA-Seq) was used for differential gene screening. Alpha and FDR use default values (Alpha=99\%, FDR=1.5). 1165 differential genes were obtained by edgeR analysis, and 676 differential genes were obtained by CORNAS analysis. The intersection of the two results was taken as the final differential gene, and a total of 656 genes were obtained (S1).

ChIP-seq data analysis

Clean reads were compared with reference genomic sequences to obtain alignment information of ChIP-seq DNA (bowtie2); alignment peak position and alignment intensity information (MACS) were found by comparing position information of reads on the genome. We use MEME-ChIP software to identify and annotate Motif, and use MEME and Dreme to detect the significant motif sequences in the peak sequence, and then use Tomtom software to compare the obtained motif sequences with known motif databases.

Other tools

Wayne maps and gene maps on chromosomes were constructed using TBtools (https://github.com/CJChen/TBtools). GO, KEGG analysis was performed using the Omic Share tools, a free online platform for data analysis (http://www.omicshare.com/tools).

\section{Results And Discussion}

In our experiments, RNA-seq analysis was first performed on HT22 cells knocked out of Pura using CRISPR/Cas9. Comparing the Pura-KO and HT22 expression profiles, we found a total of 656 differential genes (Fig. 1A). The down-regulated genes are predominant 488/656 (S1), suggesting Pur-alpha plays a major role in promoting gene expression. Pur-alpha is an important transcriptional activator, means that pur-alpha knockout has a considerable impact on many metabolic pathways involved in growth and development, such as Pathways in cancer, PI3K-Akt signaling, and cytokine-cytokine receptor interaction (Fig. 1B). In addition, we found Pura was involved in important functions such as neurotrophin signaling and Axon guidance pathways.

Pur-alpha plays an important role in the development of nerves

In order to explore the biological functions of differential genes, we performed GO annotations on the upregulated and down-regulated genes. The results showed that the down-regulated genes were involved in neuronal structure, neuronal projection, response to oxygen, and positive regulation of cellular processes (Fig. 1C). These findings highlight the vital role of Pur-alpha in the growth and development of neurons.

Pur-alpha has long been considered an indispensable factor in neurodevelopment. In experiments performed by Khalili [13], Pura knockout mice developed severe tremors, spontaneous epilepsy and other 
neurological problems at 2 weeks of age and died 4 weeks after birth. However, the specific mechanism of Pur-alpha in the early development of neurons is still unclear. In a comparative study of cerebral cortexsuspended mice at different stages after birth by Gonzalez-Lozano [16], total expression of brain proteins decreased after birth.To clarify the trend of Pur-alpha in postnatal mice, we found that Pur-alpha expression was reduced in 280-day-old mice (adults) compared to 9- and 15-day mice from Gonzalez-Lozano's study[16]. This result would suggest that the main function of Pur-alpha occurs in infancy and does not persist in adulthood. Unfortunately, although the overall difference was statistically significant, only one of the three replicates was statistically different. To explore the role of Pur-alpha in the early development of the brain, we compared the Pura-KO gene expression profile with the protein profile from Gonzalez-Lozano's study [16]. We noticed a duplication of 62 proteins (S2), suggesting that the cause of early death in Pura-ko mice may be included in these 62 proteins. We further functionalized 62 genes and found that these genes were involved in many functional and metabolic pathways (based on GO and KEGG analysis), including neuronal structure (GO:0097458), neuronal projections (G0:0043005), and nervous system. They were also involved in development (G0:0007399), neurotransmitter levels (G0:0001505), glycolysis/gluconeogenesis (mmu04066), HIF signaling pathway (mmu04066), carbon metabolism (mmu01200) (Fig. 2). As such, Puralpha is involved in the regulation of neuronal metabolism, the formation of synapses, and the establishment of projections between neurons. These changes caused by Pura knockout have a major impact on the formation of neuronal synapses and the establishment of a network of connections between neurons, so this may be the cause of premature death in Pura-ko mice.

Pur-alpha plays an important role in the progression of Alzheimer's disease

In the past few years, we have been exploring the relationship between Pur-alpha and neurodegenerative diseases, especially Alzheimer's disease (AD)[15]. In previous studies, we noticed that Pur-alpha regulates the rejuvenation of APP proteins, but the study of subsequent Pur-alpha in AD seems to be interrupted. In our study we deliberately focused on the expression of APP after Pura knockout, but unfortunately the knockout of Pura seems to have no effect on APP or APP mRNA expression. This result highlights additional complexities in AD pathogenesis. Based on RNA-seq analysis, we found that 7 genes are enriched in Alzheimer disease (Table 1,Fig. 3), and 5 genes are enriched in amyloid-beta clearance (Table 2). 
Table 1

Differential gene enriched in Alzheimer's disease

\begin{tabular}{|lllllll|}
\hline gene ID & $\begin{array}{l}\text { Gene } \\
\text { Name }\end{array}$ & $\begin{array}{l}\text { HT22 } \\
\text { (count) }\end{array}$ & $\begin{array}{l}\text { Pura- } \\
\text { ko } \\
\text { (count) }\end{array}$ & FDR & log2FC & regulated \\
\hline ENSMUSG00000015568 & Lpl & 1138 & 554 & $4.20 \mathrm{E}-05$ & -1.075796463 & down \\
\hline ENSMUSG00000018411 & Mapt & 25 & 87 & $8.02 \mathrm{E}-05$ & 1.756656016 & up \\
\hline ENSMUSG00000027820 & Mme & 361 & 86 & $7.48 \mathrm{E}-13$ & -2.105387765 & down \\
\hline ENSMUSG00000035674 & Ndufa3 & 621 & 1178 & 0.00102346 & 0.886115743 & up \\
\hline ENSMUSG00000040249 & Lrp1 & 7916 & 4904 & 0.006012088 & -0.728224954 & down \\
\hline ENSMUSG00000057666 & Gapdh & 6112 & 3641 & 0.002800884 & -0.784715839 & down \\
\hline ENSMUSG00000064358 & mt-Co3 & 1381 & 778 & 0.001266429 & -0.865192446 & down \\
\hline
\end{tabular}

Polymorphisms in the LPL gene are thought to be associated with the risk of AD [17]. LPL is a key enzyme that regulates the hydrolysis of triglycerides. LPL deficiency or dysfunction can cause dyslipidemia, which may increase the risk of $A D$ [18]. LPL binds to amyloid beta protein $(A \beta)$ and promotes cell surface association and $A \beta$ uptake in mouse primary astrocytes [19] and BV2 microglia [20]. Studies after human brain death have shown that LPL is widely distributed throughout brain tissue. Compared with control groups, LPL in the dentate gyrus granule cells and CSF samples of the AD group are significantly reduced [21]. In our study, we found similar LPL changes in AD after knocking out Pura, suggesting that Pur-alpha can regulate $L P L$, which may be a potential mechanism for AD development. Ndufa3 and mt-Co3 are mitochondria-associated proteins, which are reported relatively less in $A D$ and appear to be associated with mitochondrial dysfunction in $A D[22]$.

The large accumulation of Tau protein is one of the characteristics of AD, and Mapt is the coding gene of tau [23]. A large number of studies have shown that there is a large accumulation of Tau protein in the brain tissue of $A D$ patients. Therefore, hyperphosphorylation and deposition of Tau protein may be a cause of $A D$ [24]. In our study we found that Tau expression was up-regulated after Pura knockout, implying a potential inhibitory effect of Pur-alpha on Tau. GAPDH is a key gene in sugar metabolism, but a large number of independent studies have shown that GAPDH has non-glycolytic activity and is involved in pathogenesis and death in neurodegenerative diseases, such as Alzheimer's disease and Parkinson's disease [25]. GAPDH is often present in the AD temporal cortex along with phosphorylated tau and Ab peptides [26]. Since GAPDH has a region that binds to $A b$, some scholars believe that the aggregate of GAPDH provides seeds for the specificity of $A \beta$ [27]. Studies show that nitrosated GAPDH can enhance the degree of acetylation of $\mathrm{Tau}$; in the presence of $A b$, it can promote the aggregation of Tau into neurofibrillary tangles [28]. GAPDH and Tau appear to play highly intricate roles in the regulation of AD, so mRNA expression may not provide an adequate explanation for this phenomenon. At the same time, in our study, Tau was up-regulated and GAPDH was down-regulated. 
Table 2

Differential gene enriched in amyloid-beta clearance

\begin{tabular}{|lllllll|}
\hline gene ID & $\begin{array}{l}\text { gene } \\
\text { name }\end{array}$ & $\begin{array}{l}\text { HT22 } \\
\text { (count) }\end{array}$ & $\begin{array}{l}\text { Pura-ko } \\
\text { (count) }\end{array}$ & FDR & log2FC & regulated \\
\hline ENSMUSG00000027820 & Mme & 361 & 86 & $7.48 \mathrm{E}-13$ & -2.1053878 & down \\
\hline ENSMUSG00000024164 & C3 & 6635 & 1260 & $9.59 \mathrm{E}-26$ & -2.4339817 & down \\
\hline ENSMUSG00000040249 & Lrp1 & 7916 & 4904 & 0.00601209 & -0.728225 & down \\
\hline ENSMUSG00000005534 & Insr & 657 & 359 & 0.00126643 & -0.9090988 & down \\
\hline ENSMUSG00000023992 & Trem2 & 83 & 6 & $8.95 \mathrm{E}-12$ & -3.7995178 & down \\
\hline
\end{tabular}

Pur-alpha may affect the progression of $A D$ by regulating the expression of Ab-clearing related proteins

Decreased amyloid clearance is one of the main features of AD. In this study, we identified 5 genes involved in clearance of amyloid which may be affected by Pur-alpha. Coincidentally, the 5 genes were all downregulated. The NEP protein encoded by the membrane metallo-endopeptidase (Mme) gene is one of the major contributors to brain Ab clearance and is directly involved in the degradation of $A b$ [29]. Studies have shown that NEP inhibitors can cause biochemical and pathological deposition of $A \beta_{1-42}$ [30], and in vitro experiments show that NEP can rapidly degrade $A \beta_{1-40}$ and $A \beta_{1-42}$ [31], while exogenous supplementation of NEP can reduce the deposition of Ab in AD transgenic mice [32]. In our study, knocking out Pura resulted in a decrease in Mme expression, suggesting that the expression of NEP was dependent on Pur-alpha. Although the mechanism of this regulation is unclear, and ChIP-seq studies did not show evidence of Puralpha regulation of Mme, current research can still provide some insight. According to related studies, HIV-1 transactivator (tat) can reduce the expression and activity of NEP, thereby increasing the deposition of Ab, which is considered to be an important cause of HIV-related cognitive impairment [33]. At the same time, other studies have demonstrated the close relationship between Pur-alpha and tat. Pura promotes translation of HIV in vivo by binding to HIV-1 Tat and TAR RNA [34]. From the above studies we noticed that tat can bind to Pur-alpha, and this combination may have a similar repressive effect on Pur-alpha, causing Pur-alpha to fail to exert its normal physiological effects. The Pura knockout will result in a decrease in Mme, so the reduction in HIV-related NEP may be due to this relationship.

LRP1 is a member of the low-density lipoprotein receptor family [35], which has four extracellular ligand binding domains that bind to different ligands, including APP [36], apolipoprotein E. (Apolipoprotein E) [37], and a2 macroglobulin (a2M) [35]. LRP1 can be combined with APP before it is cut by furin [38], which slows APP movement [39], and promotes further processing of the protein [40]. LRP1 binds to APP to facilitate processing of APP, but this effect appears to increase the production of Ab [41]. Although LRP1 caused the production of $A b$, we cannot ignore the fact that it promotes Ab transport. LRP1 can directly bind to Ab through the LRP1 N-terminal domain or by binding ApoE or a2M [42]. LRP1 can transport Ab to the bloodbrain barrier by binding to $A b$ and releasing $A b$ into the blood, which is the main evidence that LRP1 is involved in Ab clearance [43-45]. In our study, Lrp1 decreased after Pura knockout, thus indicating that the 
expression of Lrp1 requires the participation of Pur-alpha, indicating that Pur-alpha plays an important role in the processing of APP and the clearance of Ab.

Insulin receptor substrates have multiple functions, including enzyme binding activity, insulin binding activity, and binding activity of insulin receptor substrates [46]. There are few studies on the involvement of Insr in Ab regulation. In a 2009 study [47], it was shown that cells with normal Insr have the ability to reduce the reduction of $A b$ oligomers to $A b$ monomers, while the Insr mutation causes a loss of this ability, leading to the aggregation of $A b$ oligomers. This increase suggests that Insr has the ability to participate in $A b$ clearance.

Complement receptor 3 (C3) plays a central role in the activation of the complement system and participates in the human immune response [48]. In the brains of Alzheimer's patients, complement components were detected in the amyloid core of senile plaques [49], and an increase in CR3 was found in microglia [50]. C3 can be cleaved by C3 convertase to form C3b. On the one hand, C3b can bind to Ab to form an Ab-C3b complex, and can bind to CR3 and activate microglia to phagocytose Ab, thereby promoting Ab clearance [51]. In another study [52], the ability of C3-deficient N9 microglia to phagocytose fibrillar $A \beta$ was significantly reduced, further confirming that activation of the complement $C 3$ system is an important factor in the phagocytosis of Ab by microglia. In our study, the decrease in Pur-alpha caused a decrease in $\mathrm{C} 3$, indicating that the synthesis of $\mathrm{C} 3$ was dependent on the presence of Pur-alpha. Therefore, while our study is based on neuronal cells, the reduction of Pur-alpha may also affect the ability of microglia.

The protein encoded by TREM2 is part of the immunoglobulin and lectin-like superfamily and is part of the innate immune system. TREM2 is a surface receptor required for microglia to respond to neurodegeneration, including proliferation, survival, aggregation and phagocytosis. TREM2 mutations cause autophagy in microglia. Increasing cyclocreatine in the diet to supplement energy can reduce autophagy of microglia and reduce Ab deposition in TREM2-deficient mice, suggesting that TREM2 affects $\mathrm{Ab}$ clearance in microglia by affecting cell energy metabolism [53]. In addition, Ab 42 deposition in agerelated macular degeneration also appears to be associated with a deficiency in TREM2 [54]; the TREM2 $\mathrm{R} 47 \mathrm{H}$ variant also shows reduced TREM2 mRNA expression and increases the risk of AD development [55]. Studies have shown that TREM2 is involved in the formation of $A D$, and in our study, there was a significant decrease in Trem2 after Pura knockout, indicating that Pur-alpha may be involved in the important process of $A D$.

The regulation of Pura on AD-related genes and Ab-cleavage-related genes appears to be indirect.

We have enriched 656 differential genes that may be regulated by Pur-alpha base on RNA-sEq. Not all genes are directly regulated by Pur-alpha. In order to clarify the regulatory mechanism of Pur-alpha on genes, we analyzed the DNA fragments that may be directly bound to Pur-alpha by ChIP-seq, and found that Pur-alpha can bind to 1389 genes (S3). To further analyze the regulation of Pur-alpha on genes, we combined ChIP-seq results with RNA-seq results, and we found that Pur-alpha can bind to 47 of them and cause a large number of changes (Fig. 4, S4). Therefore, it is believed that Pur-alpha can directly regulate 
these 47 genes, and the emergence of other differential genes may be affected by these 47 genes. Among the genes mentioned earlier in relation to $A D$ pathogenesis and Ab cleavage, only Insr is directly regulated by Pur-alpha. This means that Pur-alpha may rely on a deeper mechanism for the regulation of these genes.

We found some genes that may interact with APP and PSEN from the above 47 genes, based on string protein interaction analysis, such as Vldlr, lgfbp7, Kng2, Pros1, and Jag1. Among these results, APP, Kng2, and Igfbp7 were simultaneously regulated by phosphorylation of Fam20C enzyme [56], and there was a weak co-expression relationship between APP and Igfbp7 (co-expression score $=0.057$ ).

Vldlr belongs to the low-density lipoprotein receptor family and binds to apolipoprotein $E$ (ApoE), which is essential for Reelin pathway activation [57]. Activation of the Reelin pathway increases NMDA receptor activity by promoting tyrosine phosphorylation of the NR2 subunit, which is important in enhancing glutamatergic neurotransmission[58-61]. In addition, Reelin is involved in the transport and processing of APP, and is able to interact with A $\beta$ oligomers to antagonize its negative effects on synaptic function [6264]. In this study, we found that Pura binds directly to VIdlr DNA and positively regulates it, which means that Pur-alpha enhances Reelin activity by promoting VIdlr expression. Pros1 is a ligand for Mer tyrosine kinase (MerTK) and activation of MerTK is considered to be an important factor in amyloid-stimulated phagocytosis [65]. A decrease in Pros 1 means that the likelihood of activation of MerTK is diminished, which in turn may affect the phagocytosis of Ab. Jag1 is a substrate for BACE1 and can be cleaved by Bace1[66]. At the same time, Jag1 is a ligand of Notch that promotes the activation of Notch. Loss of BCAE1 cleavage causes an increase in Jag1, which enhances the transmission of Notch signaling. This is thought to be a possible mechanism by which BACE1 is involved in the balance of neurogenesis and astrogenesis [66]. In this study, Pur-alpha was able to directly regulate Jag1, and the lack of Pur-alpha caused up-regulation of Jag1, indicating that Pura can participate in the regulation of BACE1 on neurons and astrocytes.

\section{Conclusions}

In this study, we found a potential mechanism for Pur-alpha in neuronal development and maintenance of normal function, raising awareness of Pur-alpha. Pur-alpha's regulatory role in $A D$ was unforeseen. Our research confirms that Pur-alpha can participate in the pathogenesis of $A D$ by directly regulating the Tau and indirectly regulating $A \beta$ clearance and the regulation of $A D$-related genes. Of course, the occurrence of $A D$ is a complex process. Pur-alpha plays a vital role in the occurrence of $A D$, participating in important aspects of pathogenesis.

\section{Abbreviations}

AD: Alzheimer's disease; pur-alpha: Purine rich element binding protein A; HT22: Hippocampal neuronal cell line, HT22; GO: Gene Ontology; KEGG: Kyoto Encyclopedia of Genes and Genomes; a2M: a2 macroglobulin; ApoE: apolipoprotein E; MerTK: Mer tyrosine kinase.

\section{Declarations}




\section{Ethical Approval and Consent to participate}

Not applicable

\section{Consent for publication}

All authors have approved of the manuscript and agree with its submission.

\section{Availability of supporting data}

RNA-seq and ChIP-seq data have been uploaded to the Sequence Read Archive (SRA):

https://www.ncbi.nlm.nih.gov/sra. RNA-seq data: SRA:SUB6809189®ChIP. data: SRA:SUB6906483.

\section{Competing interests}

The authors declare that they have no competing interets.

\section{Funding}

This study was supported by the National Natural Science Foundation of China (Grant No. 81260197), Project of Ningxia Brain Plan (Grant No. 2016BZ07) and Ningxia Medical University Scientific Research Project (Grant No. XY201511). National Natural Science Foundation of China (Grants U1503223).

\section{Authors' contributions}

Jianqi Cui®Qinwen Wang $₫$ Xiaoguang Shi and Tao Sun designed research; Xiaoguang Shi囚Bingying Zhang $\rrbracket$ Shanshan Guo®Wenxin He performed research; Xiaoguang Shi囚Chengmin Yuan and Xiaofan Yang analyzed data; and Jianqi Cui \Xiaoguang Shi and Kevin Ig-Izevbekhai wrote the paper.

All authors read and approved the final manuscript.

\section{Acknowledgements}

Thanks to all the lab members who facilitated this study. 


\section{Authors' information}

${ }^{1}$ Ningxia Key Laboratory of Cerebrocranial Diseases, Incubation Base of the National Key Laboratory, Ningxia Medical University, Yinchuan, China. ${ }^{2}$ Zhejiang Provincial Key Laboratory of Pathophysiology, School of Medicine, Ningbo University, Ningbo, Zhejiang, China. ${ }^{3}$ Department of Neurology, Hongqi Hospital Affiliated to Mudanjiang Medical University $\varangle$ Mudanjiang, China. ${ }^{4}$ Perelman School of Medicine, University of Pennsylvania, Philadelphia, PA, USA

\section{References}

1. Bergemann AD, Ma ZW, Johnson EM. Sequence of cDNA comprising the human pur gene and sequence-specific single-stranded-DNA-binding properties of the encoded protein. Mol Cell Biol. 1992;12 12:5673-82; doi: 10.1128/mcb.12.12.5673.

2. Gallia GL, Johnson EM, Khalili K. Puralpha: a multifunctional single-stranded DNA- and RNA-binding protein. Nucleic Acids Res. 2000;28 17:3197-205; doi: 10.1093/nar/28.17.3197.

3. Darbinian N, Sawaya BE, Khalili K, Jaffe N, Wortman B, Giordano A, et al. Functional interaction between cyclin T1/cdk9 and Puralpha determines the level of TNFalpha promoter activation by Tat in glial cells. J Neuroimmunol. 2001;121 1-2:3-11; doi: 10.1016/s0165-5728(01)00372-1.

4. Tretiakova A, Gallia GL, Shcherbik N, Jameson B, Johnson EM, Amini S, et al. Association of Puralpha with RNAs homologous to $7 \mathrm{SL}$ determines its binding ability to the myelin basic protein promoter DNA sequence. J Biol Chem. 1998;273 35:22241-7; doi: 10.1074/jbc.273.35.22241.

5. Limesand SW, Jeckel KM, Anthony RV. Puralpha, a single-stranded deoxyribonucleic acid binding protein, augments placental lactogen gene transcription. Mol Endocrinol. 2004;18 2:447-57; doi: 10.1210/me.2003-0392.

6. Zhang Q, Pedigo N, Shenoy S, Khalili K, Kaetzel DM. Puralpha activates PDGF-A gene transcription via interactions with a G-rich, single-stranded region of the promoter. Gene. 2005;348:25-32; doi: 10.1016/j.gene.2004.12.050.

7. Lasham A, Lindridge E, Rudert F, Onrust R, Watson J. Regulation of the human fas promoter by $Y B-1$, Puralpha and AP-1 transcription factors. Gene. 2000;252 1-2:1-13; doi: 10.1016/s0378-1119(00)002201.

8. Kelm RJ, Jr., Elder PK, Strauch AR, Getz MJ. Sequence of cDNAs encoding components of vascular actin single-stranded DNA-binding factor 2 establish identity to Puralpha and Purbeta. J Biol Chem. 1997;272 42:26727-33; doi: 10.1074/jbc.272.42.26727.

9. Darbinian N, Cui J, Basile A, Del Valle L, Otte J, Miklossy J, et al. Negative regulation of AbetaPP gene expression by pur-alpha. J Alzheimers Dis. 2008;15 1:71-82; doi: 10.3233/jad-2008-15106.

10. Shelley CS, Da Silva N, Teodoridis JM. During U937 monocytic differentiation repression of the CD43 gene promoter is mediated by the single-stranded DNA binding protein Pur alpha. $\mathrm{Br} \mathrm{J}$ Haematol. 2001;115 1:159-66; doi: 10.1046/j.1365-2141.2001.03066.x. 
11. Yue F, Cheng Y, Breschi A, Vierstra J, Wu W, Ryba T, et al. A comparative encyclopedia of DNA elements in the mouse genome. Nature. 2014;515 7527:355-64; doi: 10.1038/nature13992.

12. Swinnen B, Bento-Abreu A, Gendron TF, Boeynaems S, Bogaert E, Nuyts R, et al. A zebrafish model for C9orf72 ALS reveals RNA toxicity as a pathogenic mechanism. Acta Neuropathol. 2018;135 3:427-43; doi: 10.1007/s00401-017-1796-5.

13. Khalili K, Del Valle L, Muralidharan V, Gault WJ, Darbinian N, Otte J, et al. Puralpha is essential for postnatal brain development and developmentally coupled cellular proliferation as revealed by genetic inactivation in the mouse. Mol Cell Biol. 2003;23 19:6857-75; doi: 10.1128/mcb.23.19.6857-6875.2003.

14. Zhang B, Guo S, Shi X, He W, Liu K, Sun T, et al. Pura gene knock out could deteriorate its DNA repair effect upon the genotoxic agent induced DNA damage in mouse hippocampus neuron. Chinese Journal of Cell Biology. 2018;5 40:706-16.

15. Yuan C, Chai J, Li P, Zhang B, Guo S, He W, et al. Establishment of Reporter Plasmid Containing Egr-1 Promoter and Regulatory Effects of Pura on Egr-1 Gene Expression. Journal of Ningxia Medical University. 2017;4 39:368-73.

16. Gonzalez-Lozano MA, Klemmer P, Gebuis T, Hassan C, van Nierop P, van Kesteren RE, et al. Dynamics of the mouse brain cortical synaptic proteome during postnatal brain development. Sci Rep. 2016;6:35456; doi: 10.1038/srep35456.

17. Tan L, Xing A, Zhao DL, Sun FR, Tan MS, Wan Y, et al. Strong Association of Lipid Metabolism Related MicroRNA Binding Sites Polymorphisms with the Risk of Late Onset Alzheimer's Disease. Curr Neurovasc Res. 2017;14 1:3-10; doi: 10.2174/1567202613666161027101100.

18. Xie C, Wang ZC, Liu XF, Yang MS. The common biological basis for common complex diseases: evidence from lipoprotein lipase gene. Eur J Hum Genet. 2010;18 1:3-7; doi: 10.1038/ejhg.2009.134.

19. Nishitsuji K, Hosono T, Uchimura K, Michikawa M. Lipoprotein lipase is a novel amyloid beta (Abeta)binding protein that promotes glycosaminoglycan-dependent cellular uptake of Abeta in astrocytes. $J$ Biol Chem. 2011;286 8:6393-401; doi: 10.1074/jbc.M110.172106.

20. Ma Y, Bao J, Zhao X, Shen H, Lv J, Ma S, et al. Activated cyclin-dependent kinase 5 promotes microglial phagocytosis of fibrillar beta-amyloid by up-regulating lipoprotein lipase expression. Mol Cell Proteomics. 2013;12 10:2833-44; doi: 10.1074/mcp.M112.026864.

21. Gong H, Dong W, Rostad SW, Marcovina SM, Albers JJ, Brunzell JD, et al. Lipoprotein lipase (LPL) is associated with neurite pathology and its levels are markedly reduced in the dentate gyrus of Alzheimer's disease brains. J Histochem Cytochem. 2013;61 12:857-68; doi:

10.1369/0022155413505601.

22. Wilkins HM, Carl SM, Greenlief AC, Festoff BW, Swerdlow RH. Bioenergetic dysfunction and inflammation in Alzheimer's disease: a possible connection. Front Aging Neurosci. 2014;6:311; doi: 10.3389/fnagi.2014.00311.

23. Wood JG, Mirra SS, Pollock NJ, Binder LI. Neurofibrillary tangles of Alzheimer disease share antigenic determinants with the axonal microtubule-associated protein tau (tau). Proc Natl Acad Sci U S A. 1986;83 11:4040-3; doi: 10.1073/pnas.83.11.4040. 
24. Gao Y, Tan L, Yu JT, Tan L. Tau in Alzheimer's Disease: Mechanisms and Therapeutic Strategies. Curr Alzheimer Res. 2018;15 3:283-300; doi: 10.2174/1567205014666170417111859.

25. Butera G, Mullappilly N, Masetto F, Palmieri M, Scupoli MT, Pacchiana R, et al. Regulation of Autophagy by Nuclear GAPDH and Its Aggregates in Cancer and Neurodegenerative Disorders. Int J Mol Sci. 2019;20 9; doi: 10.3390/ijms20092062.

26. Wang Q, Woltjer RL, Cimino PJ, Pan C, Montine KS, Zhang J, et al. Proteomic analysis of neurofibrillary tangles in Alzheimer disease identifies GAPDH as a detergent-insoluble paired helical filament tau binding protein. Faseb j. 2005;19 7:869-71; doi: 10.1096/fj.04-3210fje.

27. Itakura M, Nakajima H, Kubo T, Semi Y, Kume S, Higashida S, et al. Glyceraldehyde-3-phosphate Dehydrogenase Aggregates Accelerate Amyloid-beta Amyloidogenesis in Alzheimer Disease. J Biol Chem. 2015;290 43:26072-87; doi: 10.1074/jbc.M115.669291.

28. Sen T, Saha P, Sen N. Nitrosylation of GAPDH augments pathological tau acetylation upon exposure to amyloid-beta. Sci Signal. 2018;11 522; doi: 10.1126/scisignal.aao6765.

29. Miners JS, Kehoe P, Love S. Neprilysin protects against cerebral amyloid angiopathy and Abeta-induced degeneration of cerebrovascular smooth muscle cells. Brain Pathol. 2011;21 5:594-605; doi: 10.1111/j.1750-3639.2011.00486.x.

30. Iwata N, Tsubuki S, Takaki Y, Watanabe K, Sekiguchi M, Hosoki E, et al. Identification of the major Abeta1-42-degrading catabolic pathway in brain parenchyma: suppression leads to biochemical and pathological deposition. Nat Med. 2000;6 2:143-50; doi: 10.1038/72237.

31. Shirotani K, Tsubuki S, Iwata N, Takaki Y, Harigaya W, Maruyama K, et al. Neprilysin degrades both amyloid beta peptides 1-40 and 1-42 most rapidly and efficiently among thiorphan- and phosphoramidon-sensitive endopeptidases. J Biol Chem. 2001;276 24:21895-901; doi: 10.1074/jbc.M008511200.

32. Mohajeri MH, Kuehnle K, Li H, Poirier R, Tracy J, Nitsch RM. Anti-amyloid activity of neprilysin in plaquebearing mouse models of Alzheimer's disease. FEBS Lett. 2004;562 1-3:16-21; doi: 10.1016/s00145793(04)00169-3.

33. Martinez-Bonet M, Munoz-Fernandez MA, Alvarez S. HIV-1 increases extracellular amyloid-beta levels through neprilysin regulation in primary cultures of human astrocytes. J Cell Physiol. 2019;234 5:58807; doi: 10.1002/jcp.26462.

34. White MK, Johnson EM, Khalili K. Multiple roles for Puralpha in cellular and viral regulation. Cell Cycle. 2009;8 3:1-7; doi: 10.4161/cc.8.3.7585.

35. Croy JE, Brandon T, Komives EA. Two apolipoprotein E mimetic peptides, ApoE(130-149) and ApoE(141-155)2, bind to LRP1. Biochemistry. 2004;43 23:7328-35; doi: 10.1021/bi036208p.

36. Pietrzik CU, Busse T, Merriam DE, Weggen S, Koo EH. The cytoplasmic domain of the LDL receptorrelated protein regulates multiple steps in APP processing. Embo j. 2002;21 21:5691-700; doi: 10.1093/emboj/cdf568.

37. Liu Q, Zerbinatti CV, Zhang J, Hoe HS, Wang B, Cole SL, et al. Amyloid precursor protein regulates brain apolipoprotein E and cholesterol metabolism through lipoprotein receptor LRP1. Neuron. 2007;56 1:6678; doi: 10.1016/j.neuron.2007.08.008. 
38. Pietrzik CU, Yoon IS, Jaeger S, Busse T, Weggen S, Koo EH. FE65 constitutes the functional link between the low-density lipoprotein receptor-related protein and the amyloid precursor protein. J Neurosci. 2004;24 17:4259-65; doi: 10.1523/jneurosci.5451-03.2004.

39. Herr UM, Strecker P, Storck SE, Thomas C, Rabiej V, Junker A, et al. LRP1 Modulates APP Intraneuronal Transport and Processing in Its Monomeric and Dimeric State. Front Mol Neurosci. 2017;10:118; doi: 10.3389/fnmol.2017.00118.

40. Cam JA, Zerbinatti CV, Li Y, Bu G. Rapid endocytosis of the low density lipoprotein receptor-related protein modulates cell surface distribution and processing of the beta-amyloid precursor protein. J Biol Chem. 2005;280 15:15464-70; doi: 10.1074/jbc.M500613200.

41. Van Gool B, Storck SE, Reekmans SM, Lechat B, Gordts P, Pradier L, et al. LRP1 Has a Predominant Role in Production over Clearance of Abeta in a Mouse Model of Alzheimer's Disease. Mol Neurobiol. 2019;56 10:7234-45; doi: 10.1007/s12035-019-1594-2.

42. Deane R, Sagare A, Hamm K, Parisi M, Lane S, Finn MB, et al. apoE isoform-specific disruption of amyloid beta peptide clearance from mouse brain. J Clin Invest. 2008;118 12:4002-13; doi: 10.1172/jci36663.

43. Shibata M, Yamada S, Kumar SR, Calero M, Bading J, Frangione B, et al. Clearance of Alzheimer's amyloid-ss(1-40) peptide from brain by LDL receptor-related protein-1 at the blood-brain barrier. J Clin Invest. 2000;106 12:1489-99; doi: 10.1172/jci10498.

44. Pflanzner T, Janko MC, Andre-Dohmen B, Reuss S, Weggen S, Roebroek AJ, et al. LRP1 mediates bidirectional transcytosis of amyloid-beta across the blood-brain barrier. Neurobiol Aging. 2011;32 12:2323.e1-11; doi: 10.1016/j.neurobiolaging.2010.05.025.

45. Storck SE, Meister S, Nahrath J, Meissner JN, Schubert N, Di Spiezio A, et al. Endothelial LRP1 transports amyloid-beta(1-42) across the blood-brain barrier. J Clin Invest. 2016;126 1:123-36; doi: 10.1172/jci81108.

46. Fu Q, Wang Q, Xiang YK. Insulin and beta Adrenergic Receptor Signaling: Crosstalk in Heart. Trends Endocrinol Metab. 2017;28 6:416-27; doi: 10.1016/j.tem.2017.02.002.

47. Zhao WQ, Lacor PN, Chen H, Lambert MP, Quon MJ, Krafft GA, et al. Insulin receptor dysfunction impairs cellular clearance of neurotoxic oligomeric a\{beta\}. J Biol Chem. 2009;284 28:18742-53; doi: 10.1074/jbc.M109.011015.

48. Vorup-Jensen T, Jensen RK. Structural Immunology of Complement Receptors 3 and 4. Front Immunol. 2018;9:2716; doi: 10.3389/fimmu.2018.02716.

49. Eikelenboom P, Stam FC. Immunoglobulins and complement factors in senile plaques. An immunoperoxidase study. Acta Neuropathol. 1982;57 2-3:239-42; doi: 10.1007/bf00685397.

50. Strohmeyer R, Ramirez M, Cole GJ, Mueller K, Rogers J. Association of factor H of the alternative pathway of complement with agrin and complement receptor 3 in the Alzheimer's disease brain. J Neuroimmunol. 2002;131 1-2:135-46; doi: 10.1016/s0165-5728(02)00272-2.

51. Zabel M, Schrag M, Crofton A, Tung S, Beaufond P, Van Ornam J, et al. A shift in microglial betaamyloid binding in Alzheimer's disease is associated with cerebral amyloid angiopathy. Brain Pathol. 2013;23 4:390-401; doi: 10.1111/bpa.12005.

Page $14 / 20$ 
52. Fu H, Liu B, Frost JL, Hong S, Jin M, Ostaszewski B, et al. Complement component C3 and complement receptor type 3 contribute to the phagocytosis and clearance of fibrillar Abeta by microglia. Glia. 2012;60 6:993-1003; doi: 10.1002/glia.22331.

53. Ulland TK, Song WM, Huang SC, Ulrich JD, Sergushichev A, Beatty WL, et al. TREM2 Maintains Microglial Metabolic Fitness in Alzheimer's Disease. Cell. 2017;170 4:649-63.e13; doi: 10.1016/j.cell.2017.07.023.

54. Bhattacharjee S, Zhao Y, Dua P, Rogaev El, Lukiw WJ. microRNA-34a-Mediated Down-Regulation of the Microglial-Enriched Triggering Receptor and Phagocytosis-Sensor TREM2 in Age-Related Macular Degeneration. PLoS One. 2016;11 3:e0150211; doi: 10.1371/journal.pone.0150211.

55. Cheng-Hathaway PJ, Reed-Geaghan EG, Jay TR, Casali BT, Bemiller SM, Puntambekar SS, et al. The Trem2 R47H variant confers loss-of-function-like phenotypes in Alzheimer's disease. Mol Neurodegener. 2018;13 1:29; doi: 10.1186/s13024-018-0262-8.

56. Tagliabracci VS, Wiley SE, Guo X, Kinch LN, Durrant E, Wen J, et al. A Single Kinase Generates the Majority of the Secreted Phosphoproteome. Cell. 2015;161 7:1619-32; doi: 10.1016/j.cell.2015.05.028.

57. Weeber EJ, Beffert U, Jones C, Christian JM, Forster E, Sweatt JD, et al. Reelin and ApoE receptors cooperate to enhance hippocampal synaptic plasticity and learning. J Biol Chem. 2002;277 42:3994452; doi: 10.1074/jbc.M205147200.

58. Beffert U, Weeber EJ, Durudas A, Qiu S, Masiulis I, Sweatt JD, et al. Modulation of synaptic plasticity and memory by Reelin involves differential splicing of the lipoprotein receptor Apoer2. Neuron. 2005;47 4:567-79; doi: 10.1016/j.neuron.2005.07.007.

59. Chen Y, Beffert U, Ertunc M, Tang TS, Kavalali ET, Bezprozvanny I, et al. Reelin modulates NMDA receptor activity in cortical neurons. J Neurosci. 2005;25 36:8209-16; doi: 10.1523/jneurosci.195105.2005.

60. Herz J, Chen Y. Reelin, lipoprotein receptors and synaptic plasticity. Nat Rev Neurosci. 2006;7 11:850-9; doi: $10.1038 /$ nrn2009.

61. Qiu S, Zhao LF, Korwek KM, Weeber EJ. Differential reelin-induced enhancement of NMDA and AMPA receptor activity in the adult hippocampus. J Neurosci. 2006;26 50:12943-55; doi: 10.1523/jneurosci.2561-06.2006.

62. Durakoglugil MS, Chen Y, White CL, Kavalali ET, Herz J. Reelin signaling antagonizes beta-amyloid at the synapse. Proc Natl Acad Sci U S A. 2009;106 37:15938-43; doi: 10.1073/pnas.0908176106.

63. Hoe HS, Lee KJ, Carney RS, Lee J, Markova A, Lee JY, et al. Interaction of reelin with amyloid precursor protein promotes neurite outgrowth. J Neurosci. 2009;29 23:7459-73; doi: 10.1523/jneurosci.487208.2009.

64. Pujadas L, Rossi D, Andres R, Teixeira CM, Serra-Vidal B, Parcerisas A, et al. Reelin delays amyloid-beta fibril formation and rescues cognitive deficits in a model of Alzheimer's disease. Nat Commun. 2014;5:3443; doi: 10.1038/ncomms4443.

65. Jhang KA, Park JS, Kim HS, Chong YH. Sulforaphane rescues amyloid-beta peptide-mediated decrease in MerTK expression through its anti-inflammatory effect in human THP-1 macrophages. J Neuroinflammation. 2018;15 1:75; doi: 10.1186/s12974-018-1112-x. 
66. He W, Hu J, Xia Y, Yan R. beta-site amyloid precursor protein cleaving enzyme 1(BACE1) regulates Notch signaling by controlling the cleavage of Jagged 1 (Jag1) and Jagged 2 (Jag2) proteins. J Biol Chem. 2014;289 30:20630-7; doi: 10.1074/jbc.M114.579862.

\section{Figures}
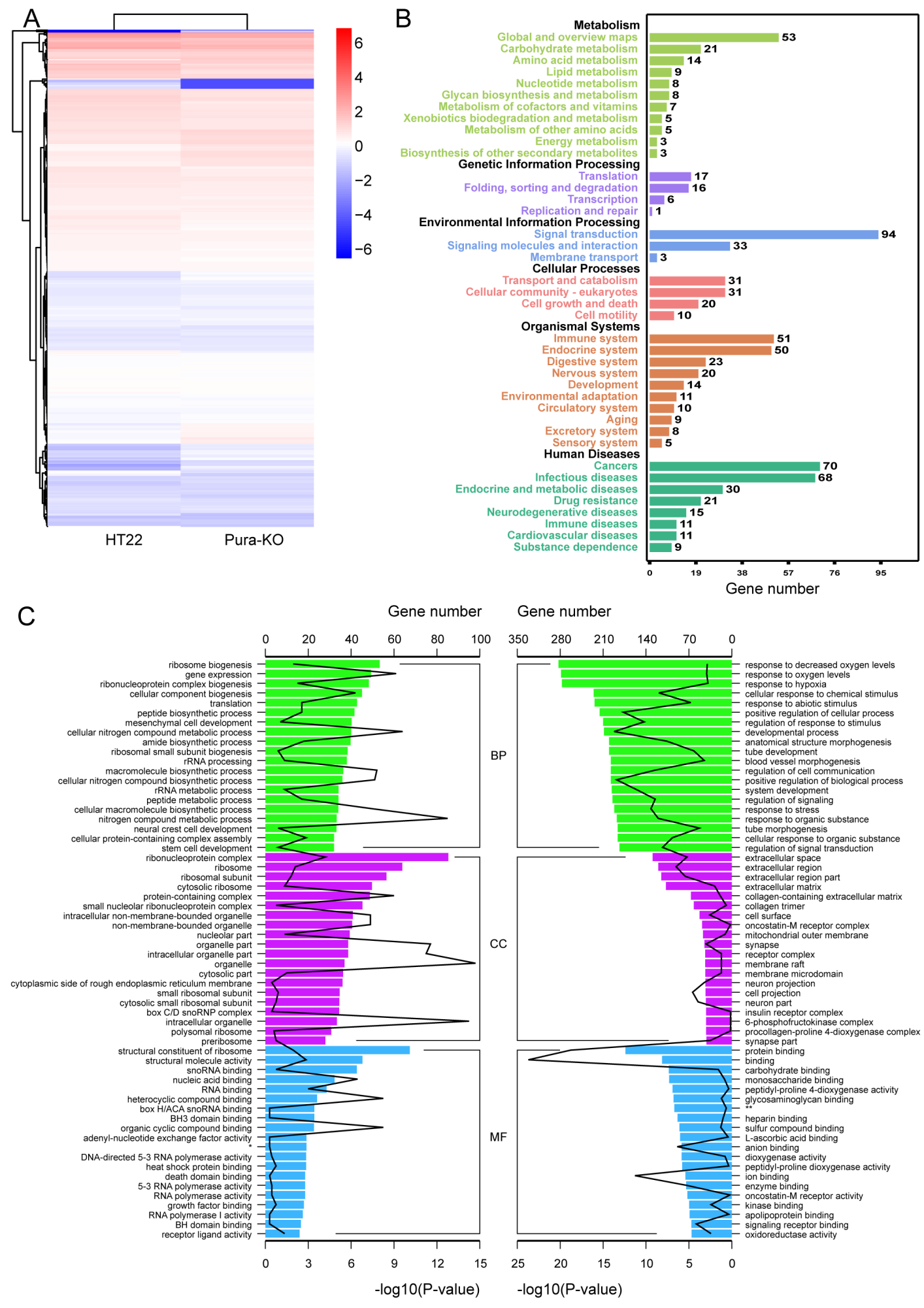

Figure 1 
Differential gene enrichment analysis. (A) The difference between the differential genes in the HT22 group and the Pura-KO group is shown in the form of a heat map. (B) Histogram results of KEGG enrichment analysis of differential genes. The abscissa is the number of genes and the ordinate is the enrichment result. (C) GO enrichment of differential genes. The left aspect is the result of up-regulated gene enrichment and the right represents down-regulation. * RNA polymerase II sequence-specific DNA-binding transcription factor binding. ** oxidoreductase activity, acting on paired donors, with incorporation or reduction of molecular oxygen, 2-oxoglutrate as one donor, and incorporation of one atom each of oxygen into both donors.
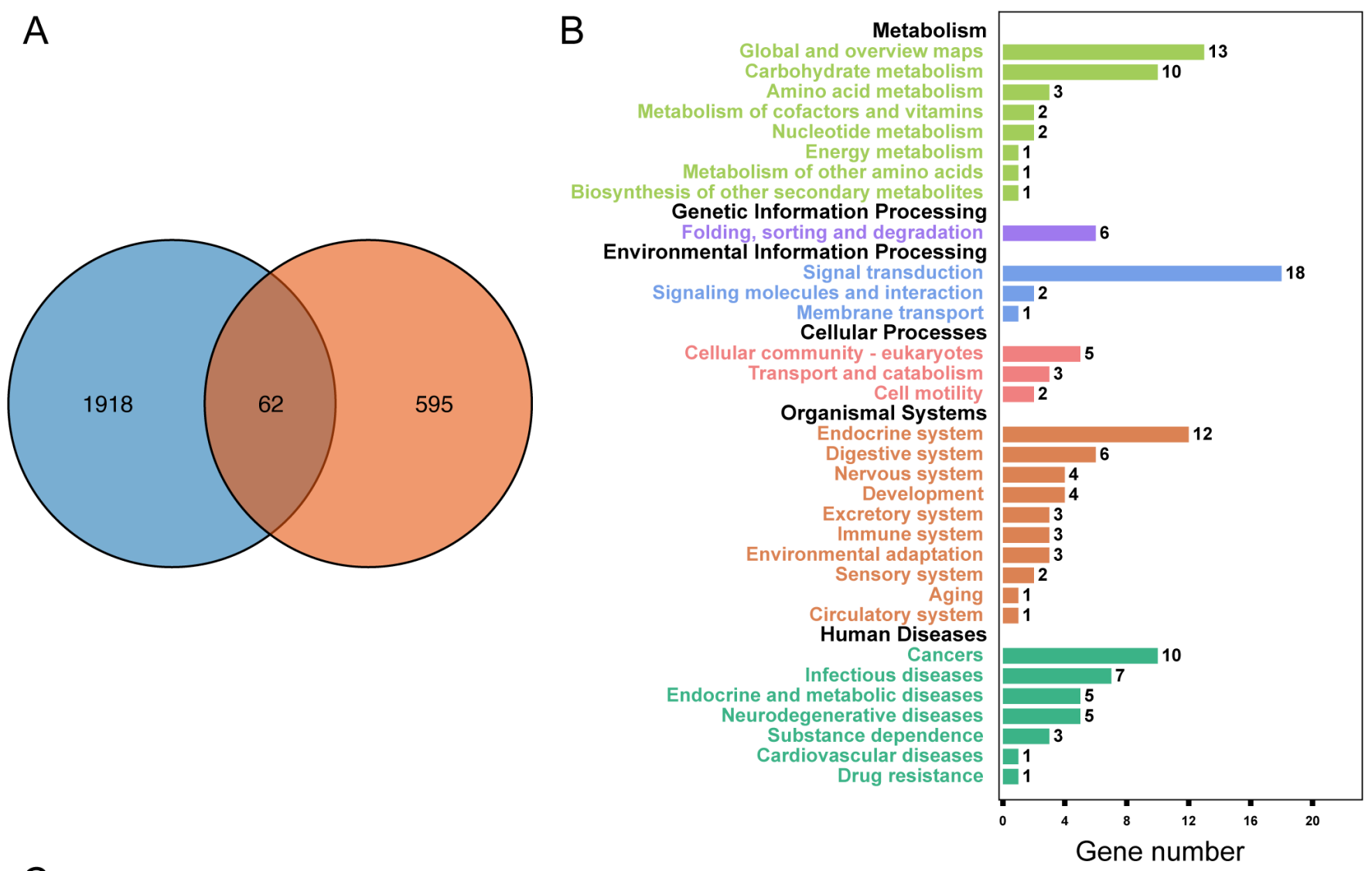

C

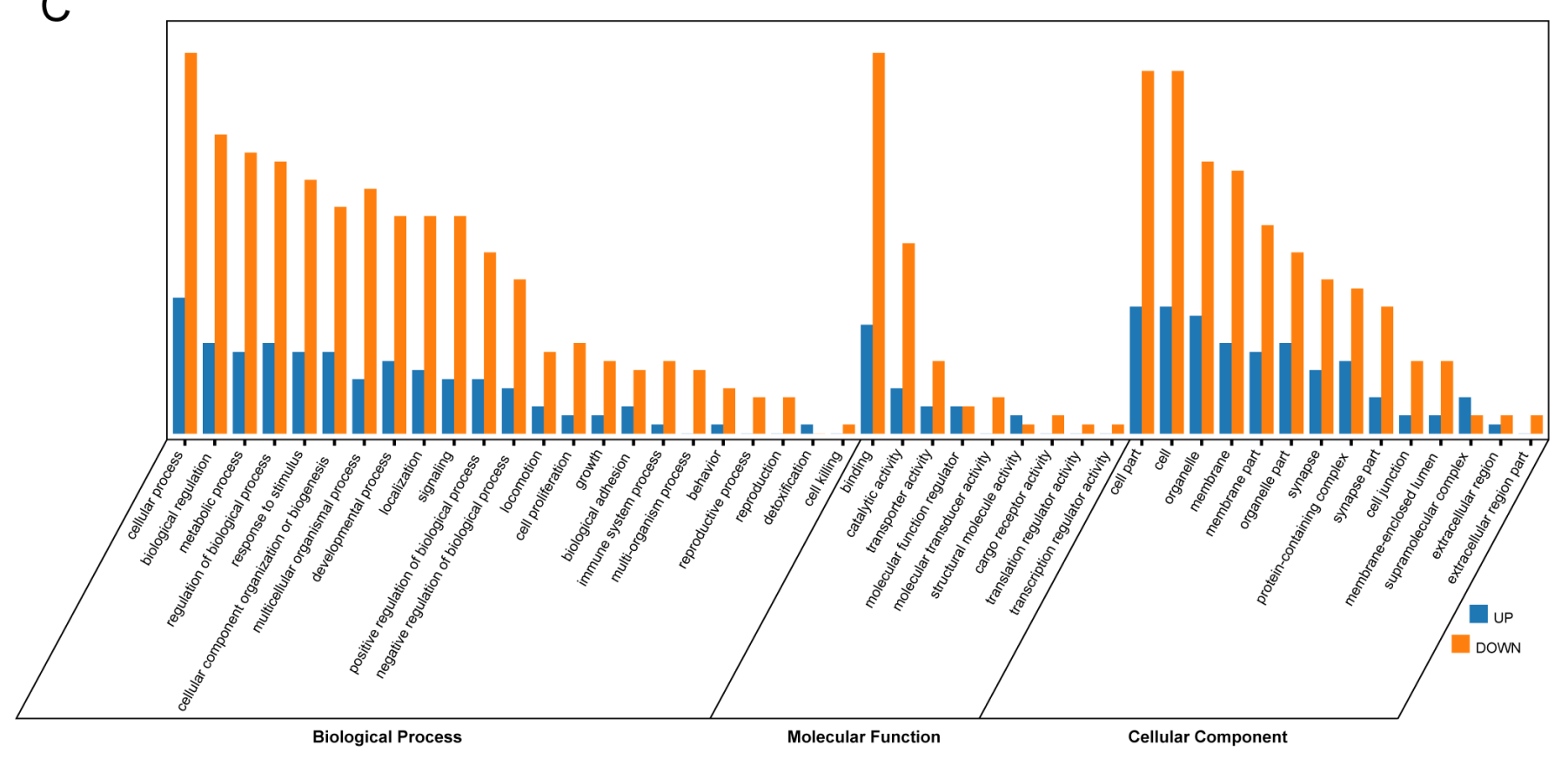

Page 17/20 
Figure 2

Comparison of early brain protein expression profiles with mice. (A) Compared with the brain protein expression profiles of mice from the 9-day postnatal experiment from the Gonzalez-Lozano study (blue), it was found that expression of a total of 62 genes appeared in the differential gene results of Pura-KO (Orange). These 62 genes may be key regulators of Pura in early brain development. (B) Histogram results of KEGG enrichment analysis of differential genes. The abscissa is the number of genes and the ordinate is the enrichment item. (C) GO enrichment of differential genes. Orange indicates genes that are up-regulated and blue indicates down-regulation.

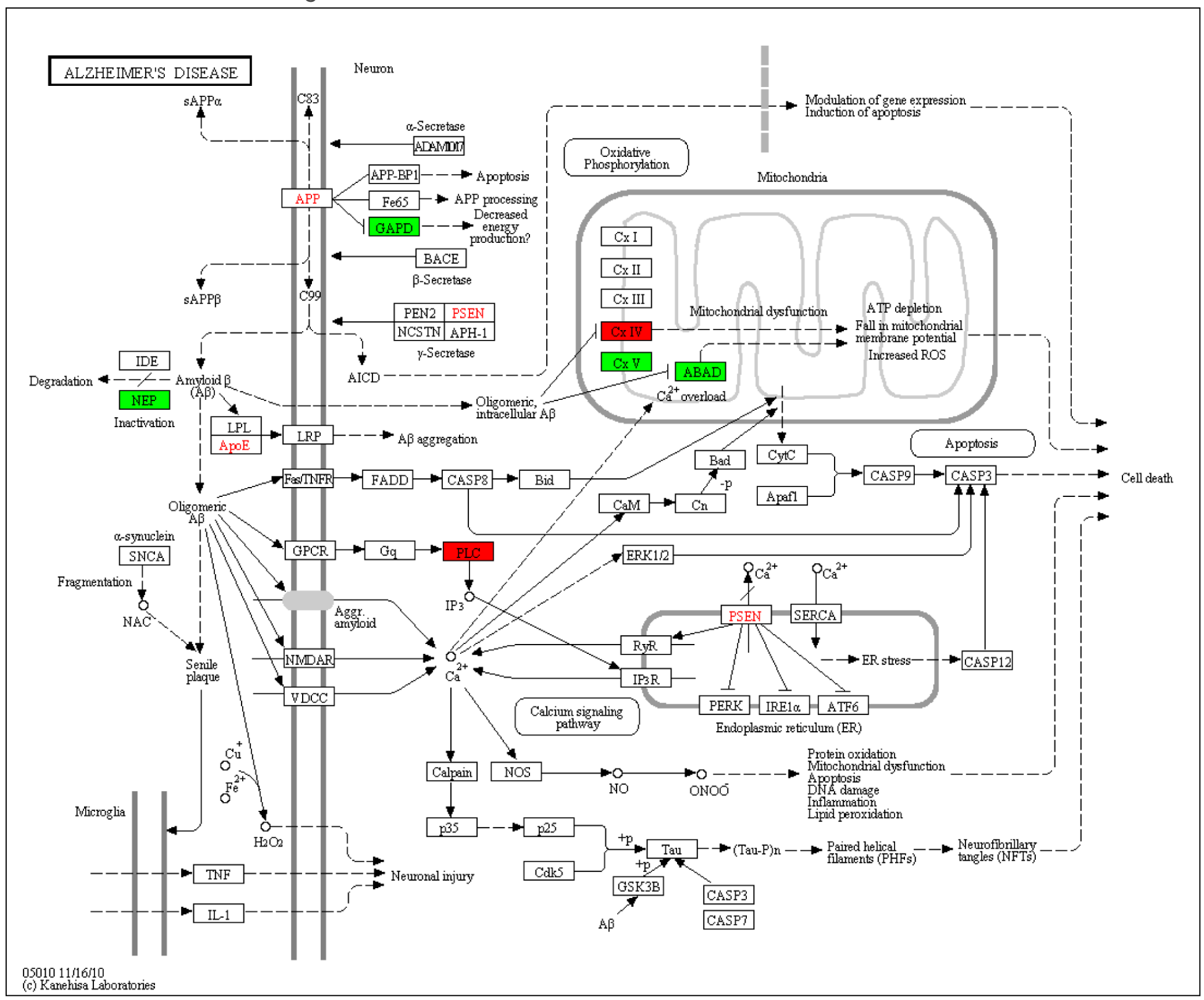

\section{Figure 3}

Distribution of differential genes in Alzheimer disease pathway. Through KEGG annotation analysis, we found 7 genes related to the Alzheimer disease pathway. There are 4 core factors in Alzheimer disease, which are APP, PSEN, ApoE, and Tau, shown in red font. The seven genes enriched were Lpl (LPL), Mapt 
(Tau), Mme (NEP), Ndufa3 (Cx I), Lrp1 (LRP), Gapdh (GAPD), and mt-Co3 (Cx IV). Green boxes indicate down-regulation of related genes, and red indicates up-regulation.

A

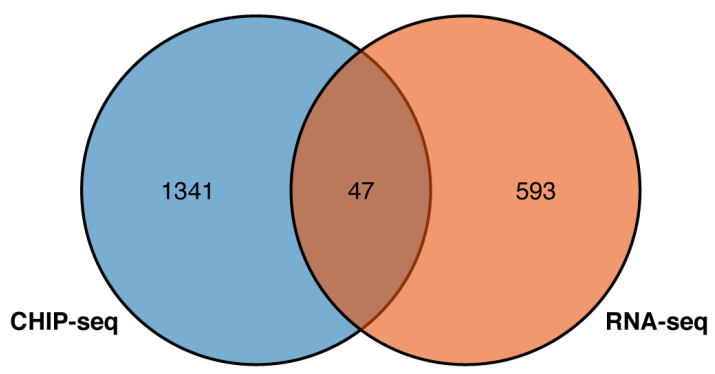

C

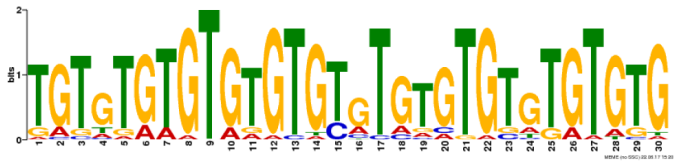

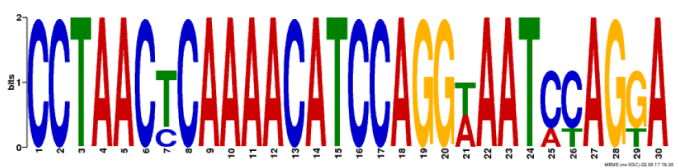

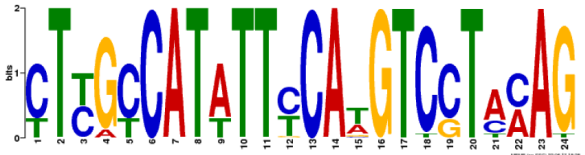

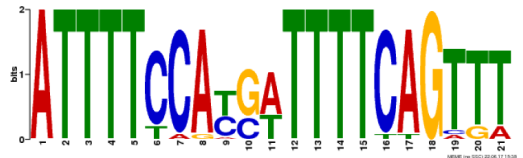

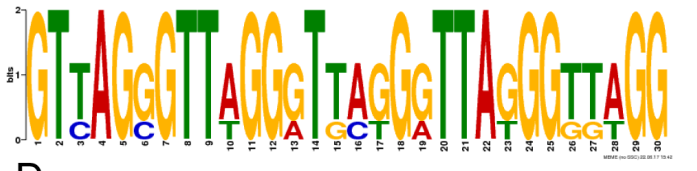

B

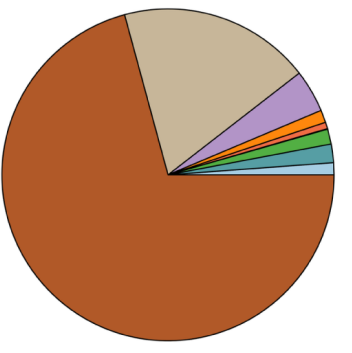

$\square$ Promoter $(<=1 \mathrm{~kb})(1.17 \%)$

$\square$ Promoter $(1-2 \mathrm{~kb})(1.81 \%)$

ㅁ Promoter $(2-3 \mathrm{~kb})(1.47 \%)$

5 5 UTR $(0.05 \%)$

Other Exon (1.22\%)

1st Intron $(4.15 \%)$

1st Intron (4.15\%)

$\square$ Other Intron (18.71\%)
$\square \quad$ Distal Intergenic $(70.79 \%)$

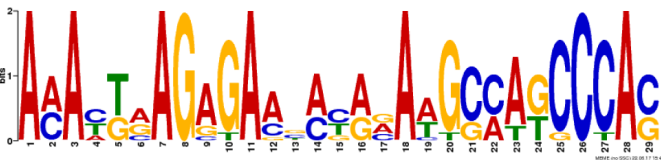
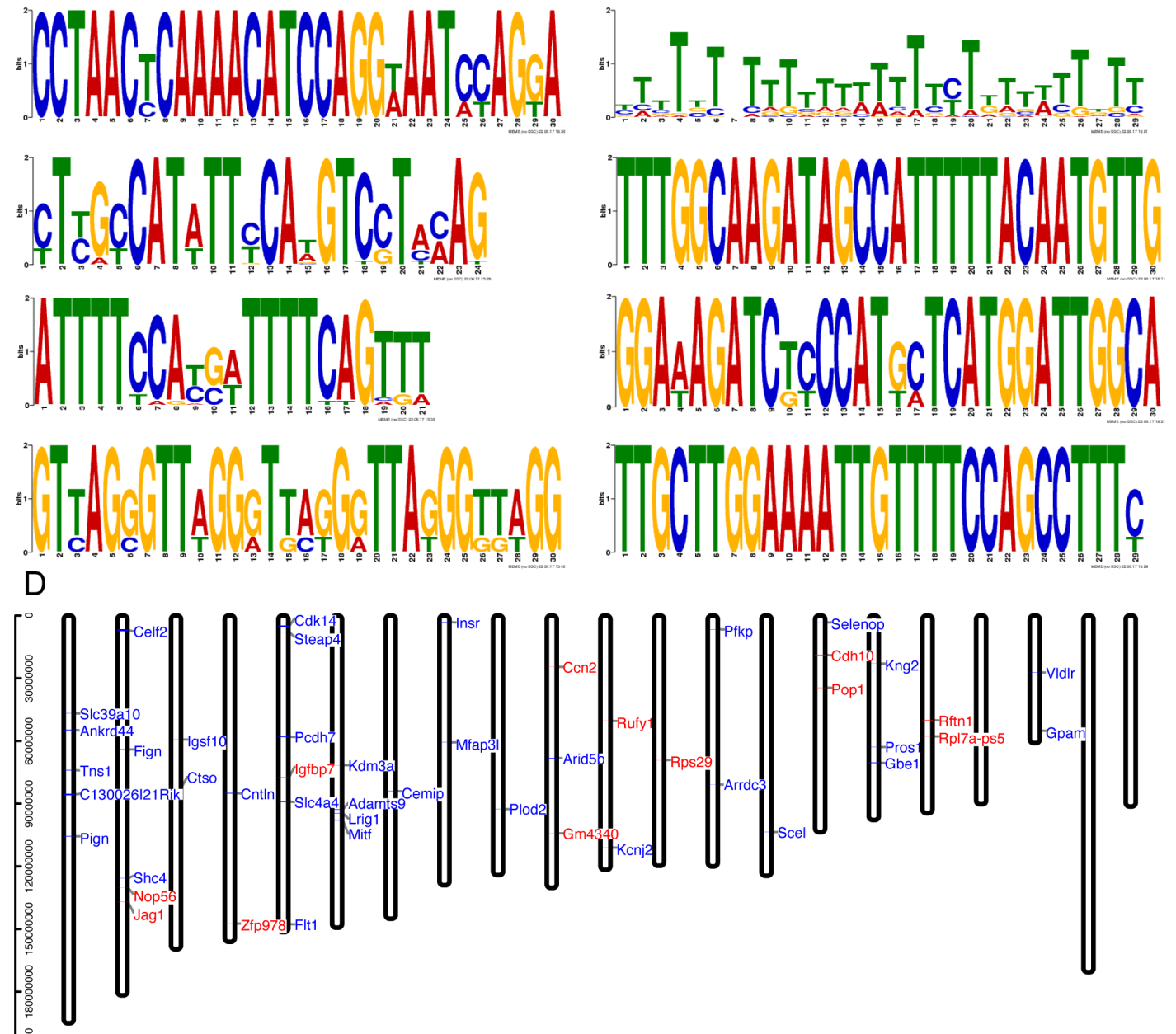

chr1 chr2 chr3 chr4 chr5 chr6 chr7 chr8 chr9 chr10 chr11 chr12 chr13 chr14 chr15 chr16 chr17 chr18 chr19 chrX chrY

\section{Figure 4}

ChIP-seq analysis. (A) ChIP-seq detected a total of 1388 genes likely to bind to Pur-alpha. Compared with 656 differential genes obtained by RNA-seq, a total of 47 genes were obtained. (B) Peak region annotation classification of Pur-alpha-bound DNA. (C) ChIP-seq analysis yields a total of 10 motifs with which Pur- 
alpha may bind, and the logos are sorted in order. (D) The position and expression of the 47 genes obtained from $A$ on the chromosome. The blue represents gene down-regulation and the red represents up-regulation.

\section{Supplementary Files}

This is a list of supplementary files associated with this preprint. Click to download.

- S4CHIPseqandRNAseqoverlapdata.xlsx

- S5qualitycontrol.xIsx

- S1Differentiallyexpressedgene.xlsx

- S3peaks.xlsx

- S2Comparedwithmousebrainproteomeat9daysafterbirth.xlsx 